\title{
树木年轮宽度与气候变化关系研究进展
}

\author{
王 婷 ${ }^{12}$ 于 丹 $^{1}$ 李江风 $^{3}$ 马克平 ${ }^{2 *}$ \\ （1 武汉大学生命科学学院, 武汉 430072) (2 中国科学院植物研究所 北京 100093)
}

(3 新疆气象科学研究所, 乌鲁木齐 830002)

摘 要 树木的生长和立地环境密切相关并受多种气候因子的影响。树木年轮宽度的增加与温度、降水、太阳辐 射、 $\mathrm{CO}_{2}$ 浓度等气候因子有着复杂的相关关系。在干旱或半干旱地区, 温度是限制树木生长的重要气候因子。生长 季开始时最低温度的升高有利于延长生长季, 与年轮宽度正相关; 但是当生长季温度过高时, 即使降水非常充裕, 当年也只能形成窄年轮。生长季的温度过高则会加快土壤蒸发失水量并提高蒸汽压差, 使土壤水分不足而不利于 树木生长，因而生长季的高温多表现为与年轮宽度的负相关。生长期内降水量与树木的径向生长也成正相关，但 当生长季的降水量充足或过多时, 降水对树木径向生长不相关或负相关。受温度和降水共同调控的土壤湿度是树 木径向生长的主要限制因子, 良好的水分状况对树木生长起决定性作用。某一地区的太阳辐射能量高常会导致高 温少雨, 故高强度的太阳辐射使表土的湿度降低而不利于树木的径向生长。而在受季风影响的地区, 树木年轮宽 度的增加与当年雨季的气候变化关系不大。当年季风到来之前的气候(温度和降水) 是树木生长的主要限制因子。 有关 $\mathrm{CO}_{2}$ 浓度的升高对树木生长的影响，研究的结果很不一致。一些温室实验及田间控制实验证明, $\mathrm{CO}_{2}$ 浓度的升 高能对短命的一年生草本植物和植物幼苗产生 施肥效应”, 并有利于其生长, 还有些研究证明 $\mathrm{CO}_{2}$ 浓度的升高能 使高海拔地带的树木年轮宽度增加, 但也有些研究认为 $\mathrm{CO}_{2}$ 浓度的升高对生长在自然条件下的自然植被影响不 大。近年来, 有关树木径向生长和气候变化的研究越来越引起人们的关注, 相关研究也取得了较大的进展。这些 研究在帮助人们了解和研究古气候变化对森林植被的影响, 以及预测未来全球变化对陆地生态系统的影响等方面 有重要的理论和现实意义。综述了气候变化对树木年轮宽度影响的研究进展和应用, 并概述了研究方法和发展前 景 希望能加快和拓宽这一领域的发展。

关键词 树木年轮宽度 气候变化 气候因子

\section{ADVANCES IN RESEARCH ON THE RELATIONSHIP BETWEEN CLIMATIC CHANGE AND TREE-RING WIDTH}

\author{
WANG Ting $^{1}{ }^{2}$ YU Dan ${ }^{1}$ LI Jiang-Feng ${ }^{3}$ and MA Ke-Ping ${ }^{2 *}$ \\ (1 College of Life Science, Wuhan University, Wuhan 430072 , China) \\ (2 Institute of Botany, the Chinese Academy of Science, Beijing 100093 , China) \\ (3 Xinjiang Institute of Meteorology, Urumqi 830002, China)
}

\begin{abstract}
There are significant correlations between tree growth and environment. Growth of trees is strongly influenced by environment and climatic factors. Tree-ring widths are significantly correlated with some climatic factors, such as temperature, precipitation, solar radiation and increasing atmospheric $\mathrm{CO}_{2}$. Temperature is found to be an important climatic factor for the growth of trees in arid and semiarid regions. The increment of the lowest temperature at the beginning of growing season can extend the growing period favoring tree growth , and significantly increase tree ring widths, while trees will have narrow rings at extreme high temperature even when accompanied with abundant precipitation in the growing season. High temperature accelerates evaporation and evapotranspiration, which can result in anomalous moisture stress for the growth of the trees. Temperature and tree ring width are inversely proportional i.e. higher the temperature, lower the tree ring width. Similarly , the precipitation in growing season also plays an important part in the growth of trees. Tree-ring widths also respond positively to precipitation at the beginning of the growing period. In contrast, they show adverse or neutral response to excessive rainfall because precipitation is no more the limiting factor to tree growth at that time. The significant relation between trees growth and climatic factors could be primarily attributed to the availability of soil moisture, which is determined by temperature and precipitation together. A favorable water balance seems to be decisive to the increment of tree-ring width. High solar radiation has a negative effect on
\end{abstract}


the tree growth , because high solar radiation usually means both low rainfall and high temperature, which in turn often result in low soil moisture and hampers tree growth. Although the rainfall is abundant in the areas influenced by monsoon, it shows no evident effect on tree-ring widths through monsoon. Pre-monsoon weather conditions (e.g. temperature and precipitation) are important limiting parameters for tree growth. Up to now , there have been no consistent conclusions about the relationship between tree-ring width and the increasing atmospheric $\mathrm{CO}_{2}$. It is confirmed that the increasing $\mathrm{CO}_{2}$ level increase growth of some tree seedlings and some short-life plant in greenhouses or in limit field experiments. Some experimental evidences also show that $\mathrm{CO}_{2}$ level can be an important limiting factor in the growth of plants in the high-altitude environment. However , there are some experiments having failed to give tree-ring evidence for a possible $\mathrm{CO}_{2}$ fertilization effect under natural environmental condition. Recently, scientists have made considerable progress in this field. These achievements can greatly help not only probe into the impact of paleo-climate on forest vegetation but also predict the effect of global climate change on terrestrial ecosystem. This paper reviewed the advances , applications and methods in this field, which aimed to widen people's knowledge and accelerate the development of this field.

Key words Tree-ring width , Climatic change , Climatic factor

树木是人类生态环境的重要组成部分，它的生 长和立地环境密切相关并受气候变化的影响, 大多 数树木年轮的宽窄能够真实地记录下每年有利或不 利的气候因素(Fritts ,1976)。从树木年轮的变化中， 我们不但可以了解树木历年的生长情况及其所在地 历年和远期气候变化的情况和规律, 还能得出气候 变化对人类赖以生存的生态系统资源的影响。因 而利用树木年轮资料获取气候与环境的变化, 不仅 成为获取过去气候环境演变数据的重要方法之一 (Fritts, 1976) ,在古气候研究中得到了广泛的应用 (Cook et al.,1991;Fritts,1991); 而且可以帮助人们 预测未来气候变化和研究气候变化对生态系统的影 响, 在全球变化研究中起着举足轻重的作用。

早在 19 世纪初或更早的时候, 就曾有一些科学 家，如法国的 Duhamel 和 Buffon、美国的 Twining 及前 苏联的 Shvedov 等, 都对树木年轮的形成进行过研 究, 尝试建立树木年轮宽窄变异与某种气候要素变 化的可能联系(吴祥定,1990)。20世纪初期 著名的 美国天文学家 Douglass (1914;1920) 就致力于在美国 西南部干旱区进行树木年轮研究。他在研究过程中 发现亚利桑那地区的树木年轮生长与当地的降水存 在着良好的对应关系, 他由此创立并推动了树木年 代学 (Dendrochronology) 的发展。树木年代学是一门 研究树木木质部各年的生长轮, 并利用年轮来定年 和评价过去环境变化的科学 (Fritts ,1976)。它是以 生理学为基础, 以树木生长特点为依据, 用来研究环 境变化影响树木生长的规律, 旨在获取气候代用资 料,重建环境变化的史实 (Fritts, 1976 ; 吴祥定， 1990)。

自从 Douglass 确立了树木年代学的原理和方法 以来, 树木年轮宽度一直是树木年代学的主要研究
对象, 且大部分的研究都是利用年轮宽度来重建过 去上百甚至几千年的降雨和温度变化, 以弥补气象 资料的不足 (Fritts, 1976; Hughes et al . , 1982 ;Stahle et al ., 1985 ; Schweingruber , 1988 ; Overpeck et al. , 1997)。本文旨在概述主要气候因子对树木年轮宽 度的影响, 并探讨相关的研究方法和最新研究进展, 以展现这一领域的研究在全球气候变化对生态系统 影响中的重要作用。

\section{1 气候变化对树木年轮宽度的影响}

Fritts (1966) 认为半干旱地区树木年轮宽度和气 候变化之间的关系可以从生物学的意义上这样进行 解释：气候变化基本上控制着树木合成养分的过程， 树木年轮宽度的不同主要是因为树木在生长季所得 到的养分和激素的量不同。在潮湿的地方或良好的 气候条件下, 树木能产生充分的养分供给生长所需， 因而能形成宽年轮。但在干燥的地方或高温、干旱 的年份 树木内部吸收营养的竞争比较激烈,养分和 激素在从树冠向根部运输的过程中因被消耗而减 少, 树干基部的形成层只能吸收到有限的养分, 这种 情况下就极易形成窄年轮。尽管树木年轮的宽度可 能与树的种类及树木所处地点和地理环境不同而 异, 但主要还是与所处大环境的降水量和温度有关。

实际上，树木生长过程中每一年年轮的形成都 取决于当年及生长前期的许多气候因子(主要是温 度和降水)的综合影响, 这种影响在树木生长和年轮 结构中是很重要的(吴祥定, 1990)。

\section{1 温度对树木年轮宽度的影响}

在干旱半干旱地区, 温度是影响树木生长的比 较敏感的气候因子 (Bhattarcharyya, et al.,1988; Borgaonkar et al. ,1999)。春季温度升高可促进形成层 
活动提早和加速阴坡的积雪融化, 从而相应地延长 植物的生长期, 有利于树木的生长 (Graumlich, 1991)。生长季的温度对树木年轮宽度的影响较为 复杂, 研究的结果也很不一致。邵雪梅等 (1991) 研 究发现, 当年的生长季是川西云杉( Picea balfouriana ) 生长旺盛的时段 较高的气温有利于光合作用， 与年轮宽度正相关。Sheppard 等 (1996), Akkemïk (2000) 以及 Mäkinen 等 (2001) 的研究证明生长季开 始时最低温度的升高有利于延长生长季, 与年轮宽 度正相关。但另有些研究发现, 当生长季的温度过 高而水分又不足时,生长季的高温多表现为与年轮 宽度的负相关 (Rolland ,1993; Larsen \& MacDonald， 1995 ;Szeicz \& MacDonald ,1996)。Gindl 等 (2000) 研 究也发现温度较低且较短的生长季不利于树木的径 向生长, 这样的生长季容易形成窄年轮。这可能是 因为生长季的高温容易加快土壤蒸发失水量并提高 蒸汽压差，从而限制了树木的生理代谢活动，不利于 树木的径向生长 (Oberhuber et al , ,1998)。Fritts (1992) 则认为生长季温度的降低常与年轮宽度负相 关或不相关, 而温度较高的生长季不但利于形成宽 年轮, 还能使晚材的密度比较大。

温度对树木生长的影响，一般表现为某年的温 度状况与当年的年轮宽度变化相对应, 但有的也能 影响到下一年生长轮的宽窄, 这在生理学上被称之 为 滞后效应” (李江风等, 2000)。上一年 8 9 月 的高温能减缓第二年的生长速率 (Rolland, 1993; Mäkinen et al .,2001)。因为这段时间的高温使土 壤含水量减少, 降水量不能满足树木蒸腾作用所需, 这样必然动用体内所储存的水分来满足需要, 并影 响下一年的生长。因此树木年轮宽度多与上一年夏 季的温度负相关 (Bräuning, 1999; Mäkinen et al. , 2001)。上一年冬季的温度状况也影响着树木第二 年的生长速率。当某年冬季温度偏低时, 使植物叶 细胞内原生质脱水, 还可能因土壤冻结而导致树木 根系冻死, 造成来年光合作用减低，且使树木生长期 缩短，从而形成窄年轮(袁玉江等, 1999)。而温暖的 冬季则可以避免叶组织冻结, 保证代谢活动正常, 从 而使树木的来年生长潜力增大 (邵雪梅等 ,1999)。 另外, 温暖的冬季还会延长生长期, 有利于树木进 行光合作用为下一年的生长积累较多的能量, 使树 木在下一生长季的径向生长加快 (Fritts, 1976 ; Gutiérrez，1991）。但当秋末和冬季的温度过高时， 树木的呼吸作用和代谢作用加强, 加速体内所储存 的以供次年生长所需的营养物质的消耗, 对植物的
次年生长不利。故树木年轮宽度常表现为与上一年 秋末和冬季的高温负相关 (Gutiérrez,1991；Rolland， 1993 ; Edwards \& Hanson, 1996 ; Cherubini et al., 1997）。

\section{2 降水对树木年轮宽度的影响}

在干旱半干旱地区, 降水也是影响树木生长的 重要气候因子。Caritat 等 (2000) 研究发现欧洲栓皮 栋 ( Quercus suber) 的形成层宽度与每月 (特别是秋季 和冬季)的降水量正相关。但一般来讲，生长期内的 降水量对树木的作用最大。许多资料证明，生长期 内降水量与树木的径向生长成正相关 (Stahle et al. , 1985 ; Archambault \& Bergeron, 1992 ; Rolland ,1993; Hughes et al. ,1994 ; Stahle et al. , 1998 ; Bräuning , 1999 ; Akkemïk，2000），这可能是由于这期间的降水 可加快光合作用产物的积累和加速植物的后期生长 (Liang et al .,2001)。Xiong 等 (2000) 还发现树木年 轮的宽窄还与生长当年夏季的降水多少成正相关。 但当生长季的降水量充足或过多而不再成为树木生 长的限制因子时, 降水与树木径向生长不相关或负 相关 (Fritts ,1992；Wimmer \& Grabner，1997)。

积雪融化的水分对树木的作用与降雨基本相 同。因雪融的速度较慢且持续的时间较长, 故降雪 对树木的作用不可忽视。在干旱半干旱地区，当春 夏季缺水时，山区积雪以融水的形式发挥了重要的 作用 (Vaganov et al. ,1999)。雪对树木生长的作用 主要体现在两个方面:1) 使前期生长得到比较完备 水分, 有利于后期生长能力增强;2）增加土壤水分 含量。当后期水分不足时, 融雪能使水分得到补 偿，使树木能够正常生长(李江风等,1984)。

降水对树木年轮宽窄的影响也具有明显的滞 后效应”。相对于温度而言，降水对树木年轮的滞后 影响要大一些。若上一年降水条件好，树体内储存 的养分就多, 次年即使降水很少, 也往往只能形成一 个正常或偏窄的年轮。但若连续 $2 \sim 3$ 年降水偏少, 则必然形成窄年轮 (李江风等, 2000)。Larsen 和 MacDonald(1995) 在研究北美白云杉( Picea glauca) 和 美国短叶松 (Pinus banksiana) 的树木年轮和气候变 化关系时发现，树木的径向生长与上一年的 7 或 8 月的降水量成正相关。这可能是由于上一年 7 或 8 月的充足降水所导致的夏季末期的土壤水分状况良 好, 使树木落叶减少且有利于树木进行光合作用并 积累营养( Rolland ,1993; Larsen \& MacDonald ,1995)。 如果上一年的秋冬季的降水量比较丰富，树体内就 有可能储存足够的水分供来年生长所需。因此, 树 
木年轮宽度还与上一年秋冬季的降水量正相关 (Fritts , 1992 ; Rolland , 1993 ; Larsen \& MacDonald, 1995 ; Oberhuber et al . 1998; Liang et al. 2001)。但 当上一年秋冬季的累计降雨量达到一定的极限值之 后，次年的年轮宽度反而与其负相关 (Caritat et al. , 2000)。

\section{3 温度和降水对树木年轮宽度的综合影响}

树木在满足一定的温度和湿度的情况下开始生 长。温度和降水常常是相互制衡, 共同作用的。例 如 积雪厚度及积雪融化的时间与冬季的降水和夏 季的温度都有关系。冬季较多的降水利于降雪积 累, 而夏季温度升高则可加速积雪融化和加快树木 形成层的活动 (Groisman et al , 1994)。Peterson 和 Peterson (1994) 在研究气候变化与生长在美国北卡 斯克德山脉的亚高山针叶树木径向生长与气候变化 的关系时, 发现气候变化对亚高山森林的影响主要 依赖于冬季积雪的积累厚度和春季雪融化的速度。 春季积雪融化时间提前和较暖的夏季有利于北部山 坡树木的生长，与树木年轮宽度成正相关，但却有可 能加快山脊和南坡上夏季的干旱频率并使夏季的干 旱程度更加严重, 从而加剧本已存在的土壤湿度的 不足并限制树木的生长，与年轮宽度负相关 (Peterson \& Peterson ,1994 ;Vaganov et al. , 1999)。

在干旱半干旱地区, 受温度和降水共同调控引 起的土壤湿度是树木年轮径向生长的主要限制因子 ( Tessier et al . , 1989 ; Szeicz \& Macdonald ,1996 ; Borgaonkar et al., 1999; Bräuning, 1999$)$ )。Graumlich (1991)认为, 温度和降水的综合作用对树木生长的 影响大致可分为以下 3 种类型 : 1) 干旱的胁迫。高 温和低降水量的联合作用引起的干旱提高了水分胁 迫并由此降低了光合作用 (Fritts et al.,1965a)，不利 于储存光合作用产物以供树木生长所需。2) 短而 冷的生长季的交互作用。生长季中低温和高降水量 的联合作用 (如厚且持久的积雪) 使光合作用降低 (Fritts ,1976) ，不利于树木生长。3) 温度-降水的相 互作用。低降水量的冬季引起的干旱胁迫以及高降 水量的冬季所引起的低温都会限制树木的生长 (Graumlich ,1991)。

受温度和降水共同调整引起的土壤湿度对树木 生长的影响具体表现为: 当降水量低于一定值时, 温 度的高低和树木年轮宽度的生长呈现明显的负相关 性, 而且降水量越少这种负相关性越显著; 在降水适 宜的情况下, 温度对树木生长的影响不明显; 而在 降水高于某一极限值时, 随着温度的上升 树木的生
长达到某一最高值, 而后温度的升高对树木生长的 影响不大(张志华等,1996)。这可能是因为, 在温度 满足树木生长的情况下, 温度升高 增加林区的蒸发 量, 降低森林的土壤含水量，从而抑制树木的生长。 且这种影响在降水稀少的情况下更为明显。而在降 水适宜的情况下，树木本身对降水的正需求和因温 度增高而导致森林土壤含水量的降低所产生的负影 响, 在某种情况下相互持平。当降水比较充分时, 降 水不再是树木生长的限制因子，树木对温度的需要 则明显地表现出来。

\section{4 太阳辐射对树木年轮宽度的影响}

一般情况下, 太阳辐射能量高常会导致高温少 雨。高强度的太阳辐射能抑制树木的生长 (Oberhuber et al .,1998; Liang et al. 2001)。当树木在当年 生长季节需要适宜的温度和充分的降雨量时, 高强 度的太阳辐射使表土的湿度降低而不利于植物根系 吸收土壤肥力, 结果必然减缓树木的生长 (Rolland, 1993 ; Oberhuber et al. ,1998)。例如, 适于在湿地环 境中生长的柏树即使在土壤水分饱和的状况下, 如 果遭受到强烈的太阳辐射和高温, 也有可能引起干 旱, 不利于树木生长, 以致形成窄年轮。这主要是因 为强烈的太阳辐射使树木的蒸腾作用速率超过吸水 速率，导致蒸汽压的不连续，使水分不能满足树木生 长需要 (Rolland ,1993)。

另外，太阳辐射能量的高低还与坡度、坡向等环 境因子有关 (Rolland,1993)。Oberhuber 等 (1998) 还 发现山体的南坡较北坡吸收的太阳辐射能量高, 因 而导致南坡春季早期的干旱程度要比北坡严重得 多。这种现象或许可部分地解释树木年轮的宽窄与 坡度和坡向的相关关系。

\section{5 季风对树木年轮宽度的影响}

在受季风影响的地区, 当年季风到来之前的气 候(温度和降水) 是树木生长的主要限制因子, 树木 年轮宽度的增加与当年雨季的气候变化关系不大。 季风对树木生长的影响也主要体现在由季风所引起 的温度和降水的变化对树木生长发挥作用, 由温度 和降水共同决定的土壤湿度是决定树木生长的主要 限制因子(Borgaonkar et al ., 1999 ;Bräuning, 1999)。

例如，在受西南季风影响的喜马拉雅山西部地 区, 降水集中在受季风影响的雨季, 树木年轮宽度的 增加与当年雨季的气候变化关系不大。雨季到来之 前的几个月 ( $3 \sim 5$ 月) 的气候 (温度和降水) 是树木 生长的主要限制因子。其中, 树木年轮宽度跟降水 正相关, 与温度负相关。这些地区从 3 月起温度开 
始上升 到雨季来临之前的 5 月达到生长季的最高 温，但在这个季节的降水却很稀少。这几个月的高 温加快了蒸发,由此引起的蒸发和蒸腾失水导致土 壤湿度的降低而不利于树木的生长 (Borgaonkar et $a l$ ，１999）。这个季节的少量降水将有利于树木年 轮的生长。5 月之后, 由于雨季的到来, 温度不再是 树木生长的限制因子 (Borgaonkar et al. , 1999)。

而在不受季风影响的地方, 主要是由温度和降 水共同调控的土壤湿度对树木生长的过程起着非常 重要的作用。这些地方一年中的 $5 \sim 8$ 月的温度最 高, 降水稀少。由温度决定的土壤湿度是树木生长 的限制因子，与树木年轮宽度负相关。这几个月中 的少量降水能加快树木的径向生长, 并与其正相关 (Borgaonkar et al. 1999)。

\section{$1.6 \mathrm{CO}_{2}$ 浓度对树木年轮宽度的影响}

上一世纪以来, 由于人类活动的加剧使地球上 的 $\mathrm{CO}_{2}$ 浓度不断上升 (Woodwell et al , 1983)。大气 中不断上升的 $\mathrm{CO}_{2}$ 浓度直接影响植物的生长、生产 力和净光合作用(Lemon ,1983; Emanuel et al. 1985 ; Solomon , 1986 ; LaDeau \& Clark , 2001)。对于 $\mathrm{CO}_{2}$ 浓 度的升高对树木生长的影响, 人们得出的研究结果 较不一致。

LaMarche 等 (1984) 以及 Graybill 和 Shiyatov (1989) 提出 $\mathrm{CO}_{2}$ 浓度的升高可加快高海拔地带树木 的径向生长。他们在研究美国加利福尼亚南部干旱 地区和科罗拉多州洛矶山脉的亚高山森林上限 (3 $100 \mathrm{~m}$ ) 的几种松树 ( Pinus longaeva, P. aristata, P. flexilis) 的生长与气候变化的关系时发现,1950 1980 年间的树木生长与气候变化没有明显的相关。 他们因而提出假设: 可能是因为空气中不断增加的 $\mathrm{CO}_{2}$ 浓度引起的 施肥效应” 促进了亚高山针叶树的 径向生长 (LaMarche et al. ,1984a; Graybill \& Shiyatov，1989)。人工控制的温室及田间试验证明了 $\mathrm{CO}_{2}$ 浓度的升高可加快高海拔地带植物的径向生长 (Green \& Wright ,1977 ; Rogers et al. , 1983 ; Ceulemans \& Mousseau, 1994 ; Körner \& Diemer , 1987)。 Mooney 等 (1964) 在实验室的试验则证明 $\mathrm{CO}_{2}$ 浓度的 降低可限制有些植物的径向生长。另有一些研究也 发现 $\mathrm{CO}_{2}$ 浓度的升高可加快高海拔自然植被的径向 生长( Nicolussi et al.,1995)。

但以上实验多是一些控制实验且研究对象大部 分是一年生植物, 对多年生植物的研究通常只限于 很短时间的观测。另有些科学家的研究发现自然环 境下树木生长和 $\mathrm{CO}_{2}$ 浓度之间并没有确定的联系。
如 Kienast 和 Luxmoore (1988) 研究发现在科罗拉多 州洛矶山脉高海拔处的树木年轮宽度变化与 $\mathrm{CO}_{2}$ 的 施肥效应成负相关。Graumlich(1991) 也发现加利福 尼亚州内华达山脉针叶树的径向生长与 $\mathrm{CO}_{2}$ 浓度的 升高没有直接的关系。而 Jacoby 和 D'Arrigo (1997) 则认为 $\mathrm{CO}_{2}$ 浓度升高对自然生态系统树木径向生长 的施肥效应是非常有限的。

虽然大量的研究工作试图评价大气 $\mathrm{CO}_{2}$ 浓度升 高和变暖是否有利于植物的生长, 但目前尚无定论 (Ceulemans \& Mousseau,1994; 蒋高明等,1997)。植 物对气候变暖和 $\mathrm{CO}_{2}$ 浓度升高的反应，既与树种的 不同及降水量的多少有关, 也与树木所处的海拔及 土壤水分和土壤营养等环境条件密切相关 (Graumlich, 1991;Ceulemans \& Mousseau，1994)。因此, 研究 自然环境条件下的 $\mathrm{CO}_{2}$ 浓度的增加及由温室效应所 导致的大气温度的升高对生态系统的影响是当前生 态学研究的焦点问题之一。研究时如能将 $\mathrm{CO}_{2}$ 浓度 与温度及降水变化结合起来综合考虑其对树木生长 的影响 将有望得出较好的结果。

\section{7 其它气候因子与树木年轮宽度的关系}

Fritts 等 (1965b) 提出树木年轮生长状况沿生态 梯度变化而不同。后来 LaMarche (1974a; 1974b) 及 Kienast 等(1987)的研究则表明在亚高山地区, 由坡 度、坡向等地形条件决定的温度、湿度等条件是决定 树木径向生长的主要因素。LaMarche 等 (1984b) 研 究发现火山喷发可引起霜冻轮的形成, Yamaguchi 等(1997)还发现地震对树木年轮的宽度也有一定的 影响。还有些研究表明树木的种类不同, 对气候变 化的响应特征也不相同 (Becker ,1989; Peterson \& Peterson ,1994; Tardif \& Bergeron ,1997; Xiong et al. , 2000)。随着人们对气候变化和树木年轮形成原因 和结构特点认识的加深, 有关气候变化与树木径向 生长关系的研究范围将越来越广泛。

\section{2 研究方法及进展}

树木年轮与气候变化之间关系的研究是一项十 分复杂的工作, 主要是基于木质部生长层变化能够 响应环境条件这样的事实, 依据树木年轮气候学和 数理统计学等基本原理, 并通过年轮宽度等生长量 与各种气候因子的相关分析, 来进行树木年轮与气 候变化的关系及其应用方面的研究。其中, 最初主 要是利用年轮量测仪器与计算机联机使用, 从而得 到树木年轮宽度值（吴祥定，1990），再根据所测得的 树木年轮宽度数据建立树轮年表并结合现有气象资 
料来重建过去的气候和环境变化。但重建的精度和 可靠性在很大程度上取决于树木年轮定年的准确性 和能否最大限度地剔除非气候环境因子对树木生长 的影响。20世纪 80 年代初, Meko (1981)、Cook (1985) 等提出了用时间序列模式消除树木生长持续 性和树木相互竞争产生的非同步扰动在气候重建过 程中的影响的方法, 在干旱、半干旱地区或森林上 限附近等特殊生境研究的基础上, 使在湿润地区和 森林内部的研究取得了较大的进展 (Wiles et al. , 1996 ;Jacoby \& D’ Arrigo ,1997)。

20 世纪 60 年代, X 射线技术成功地应用于树木 年代学研究中, 并发展了以木材密度为环境代用资 料的测量技术 (Polge ,1970)。木材密度作为树轮细 胞直径及木质部细胞壁厚度和细胞腔大小等结构的 间接反映，它包含了从年轮宽度中无法提取的环境 信息 ( Parker \& Henoch , 1971 ; Wimmer \& Grabner, 2000 ;D’ Arrigo \& Jacoby ,1999)。但由于密度分析所 用的仪器相当昂贵，操作过程也比较繁琐，目前这方 面的研究主要集中在欧美几个国家的树木年轮实验 室中。

随着年轮密度越来越受树木年代学家的重视， 便产生了用图像分析技术获取图像亮度资料的研究 (Thetford et al，1991)。图像分析把树木年轮对可 见光的反射程度作为指标, 研究指标变化与气候变 化之间的关系。其物质基础是年轮细胞大小和细胞 壁厚度受环境因子影响而显示的差异 (邵雪梅, 1997)。由于细胞大小和细胞壁厚度会直接影响年 轮灰度, 可以从年轮灰度中提取气候变化信息(刘洪 滨等, 1996 邵雪梅,1997)。利用图像分析能同时得 到年轮宽度、早材宽度、晚材宽度、早材反射亮度、晚 材反射亮度、整个轮的平均亮度等 8 个指标(邵雪 梅,1997)。研究证明, 采用图像分析系统与树轮宽 度量测仪两种不同的途径得到的年轮宽度系列基本 相同, 说明图象分析结果在树轮气候学分析中是可 信的(刘洪滨等,1996)。图像分析是继 X 射线密度 分析之后的树木年代学研究技术上的又一大进步, 这项技术的应用使计算机系统代替价格昂贵与繁杂 的密度分析仪, 在不影响分辨率和数据质量的前提 下, 大大减轻了劳动量, 降低了实验成本(Thetford $e t$ al. 1991 汶洪滨等, 1996 ;Sheppard et al. 1996 邵雪 梅, 1997)。

先进的树轮分析仪器 Dendro-2003 densitometer 和 WinDENDRO 的发明和应用，使得图像分析技术 获取图像资料的研究水平也相应有所提高。这两种
仪器都是在基于 $X$ 射线技术的基础上，利用高分辨 率的光扫描仪代替摄像机, 通过平板扫描仪直接获 取树轮信息, 并运用计算机手段和数字图像技术对 树轮图像进行分析处理, 从而得到所分析样品的年 轮密度或宽度值。与普通的照相机和摄像机相比， 利用高分辨率扫描仪可直接接触树轮样本, 无需额 外的光学系统，避免了放大率非线性失真问题; 同 时 稳定的冷阴极灯管也克服了因光源波动而产生 的干扰，并使得图像分析技术在质量上和精度上可 以满足树木年代学研究的需求(谢昆青等,2000)。 利用 Dendro-2003 densitometer 和 WinDENDRO 仪器不 仅能分析出年轮的密度, 还能同时得到年轮宽度及 早、晚材宽度和早、晚材密度以及它们分别占全年年 轮的总宽度和总密度的百分比等多项指标, 这样就 大大地节省了时间。但由于 $\mathrm{X}$ 射线密度分析法对 切片样本的处理有很高的要求, 并且需要多次转换 及复杂的维护管理，一般情况下,WinDENDRO 多用 来测量树木年轮的宽度。Savva 等(2002) 利用 Dendro-2003 densitometer 研究了种植在中西伯利亚的南 部针叶树林带的欧洲赤松 (Pinus sylvestris) 的年轮， 成功地得到了不同起源的欧洲赤松年轮中遗传基因 和环境条件的关系。Abrams 等 $(1997 ; 1998)$ 也利用 WinDENDRO 成功地研究了气候变化与树木年轮的 关系。但是，对于那些除了早晚材颜色变化以外，还 有其它因素可能引起颜色变化的树种 或心材、边材 有明显颜色变化的树种, 图像技术就无法代替密度 分析(邵雪梅,1997)。

近年来, 为了在有限的树轮宽度资料中提取更 多的气候变化信息，并使根据宽度分析的结果更加 可靠, 其它学科的方法不断被引用到树轮气候学研 究中来(邵雪梅, 1997)。例如, 卡尔曼滤波方法被用 来描述树木生长对气候要素在不同时段的不同响应 （Steele \& Fiedler ,1996) 䏨应面分析方法被用来同时 重建温度和降水两个变量 (Graumlich,1991) ; 贝叶斯 方法被用来展示重建结果的可靠性 (Van Deusen \& Reams ,1996)。另外,20世纪 80 年代末兴起的模式 研究在树木年代学研究中的应用, 保证了利用相关 关系对气候要素重建的可靠性(邵雪梅,1997)。与 此同时, 新的技术也不断地被引用到树轮分析中来。 这些技术的成功引用并辅以数学分析和计算机技 术, 为树轮宽度与气候变化关系的研究开辟了新的 领域,并显示出广阔的研究前景。

\section{3 应用研究}

树木年轮结构对气候变化的响应的研究，树木 
年轮学最初的研究目的就是通过年轮宽度的变化规 律找出气候变化的规律。自从 Douglass 确立了一套 完整的从取样到定年进而了解过去环境变迁的树木 年轮学的基本原理和方法以来, 利用树木年轮宽度 来推断环境的气候变化一直是树木年代学研究的一 个主要方面。目前, 世界各国均在利用树木年轮宽 度重建古气候变化的研究方面, 无论是从长时期气 候要素的重建还是某个短时期的甚至 1 个月以内的 气候变化的认识(Byram \& Doolittle，1950 ; Mäkinen et al . ,2001) 都取得了可喜的成绩。其中, 英国成 功地重建了爱丁堡 1721 1975 年间的温度( Hughes, 1984) ; 美国重建了 1000 多年来的温度 (Graybill \& Shiyatov, 1989; Graumlich,1993) 和降水 (Graumlich , 1993）; 芬诺斯堪迪亚地区自从公元 500 年到 1990 年间的共 1400 年的夏季温度也得到了成功的重建 (Briffa et al . 1990)。在中国，利用树轮宽度资料重 建了陕西镇安 200 多年来的初春温度 (刘洪滨等, 2000 ) 和陕西秦岭 300 多年来的季节降水 (Hughes et $a l$, 1994), 以及乌鲁木齐河 360 年的径流量变化 (李江风等,1997)和青海都兰 1835 多年来的气候变 化(康兴成等, 1997)。

大范围的森林虫害、火灾、旱灾等不利因素对树 木生长的影响都能在年轮结构和年轮宽度的变化模 式上反映出来 (Schweingruber，1996)，因而，可利用 树木年轮宽度特征来分析和重建森林干扰历史 (Fritts ,1976 ; Schweingruber, 1988 ; Veblen et al. , 1991a;1991b; Nowack \& Abrams, 1997 ; 夏冰等, 2001)。近年来, 通过利用树木年轮序列中提取的信 息来重建森林干扰的应用研究受到了广大生态学家 的重视, 相关研究范围越来越广泛, 如干旱 (Jenkins \& Pallardy,1995)、涝灾 (Holmes et al。,1979)、虫害 ( Veblen et al.,1991b)、火灾 (Swetnam,1993)、火山爆 发(LaMarche \& Hirschboeck,1984b) 等干扰事件都得 到了很好的研究和重建。另外, 通过利用树木年轮 资料, Jones 等(1984)还研究了英国南部的河流量变 化 Schweingruber(1988)和 Hughes 等(1982) 也研究了 当地的极端气候变化状况。McClenahen (1995) 则概 括阐述了如何利用树木年轮分析的方法来恢复森林 的干扰历史。

随着人类活动的加剧和现代工业的发展，以及 伐木毁林的不断增加, 大气中的 $\mathrm{CO}_{2}$ 等温室气体也 日趋升高。研究温室气体浓度增加及由温室效应所 导致的大气温度的升高对生态系统的影响是当前生 态学研究的热点之一。近年来, 随着全球变化及其
对陆地生态系统的影响日益受到关注，树木年轮资 料由于定年准确、连续性强和分辨率高等特点, 利用 年轮资料来研究和预测环境因素 (尤其是气候因素) 对树木生长和森林结构与功能影响的研究也逐渐受 到重视(Johnson \& Fryer ,1989; 兰涛等, 1994 ;Szeicz \& MacDonald ,1994 ;王水等,1995 ;Jacoby et al . , 1997)。 这些研究在帮助人们了解和研究古气候变化对森林 植被的影响, 以及预测未来全球变化对陆地生态系 统的影响等方面有重要的理论和现实意义。

另外，虽然作物的产量与树木的生长之间没有 直接的联系，但无论作物的产量还是树木的生长都 与同一地区的环境变化紧密相关。这样, 作物的产 量和树木年轮宽度之间可能存在某种潜在的联系 (梁尔源，2001)。能否利用普遍存在的树木年轮来 分析或预测作物产量, 显然是一件值得尝试的有价 值的工作。但这类研究在国际并不多见。袁玉江 (1987)认为, 当年轮与作物产量受相同的主要气候 限制因子制约时，可用年轮来延长作物气候产量系 列, 并由此外推可得到作物气候产量的多年预报值。 $\operatorname{Vaganov}(1990)$ 提出的用年轮宽度序列预测作物产量 的方法, 以及 Vaganov 和 Kachaev (1992) 提出的利用 树木年轮重建小麦产量的方法等，对用树木年轮宽 度变化来研究当地的作物产量具有一定的指导作 用。夏冰等 (1994)通过对江苏溧阳马尾松年轮的分 析，与当地的作物平均产量建立回归模型，不仅较好 地再现了过去 116 年的作物产量的变化趋势, 而且 还预测了末来短期内的作物产量的波动情况。这 样，通过同一地区的树木生长和作物产量与环境变 化之间的关系, 进而利用年轮宽度来重建作物产量 是一项非常值得尝试的研究, 也使树木年代学的应 用范围得到进一步的拓宽。随着人们对自然环境的 认识和研究的加深, 有关树木年代学的应用范围将 会越来越广泛。

\section{4 研究前景}

利用树木年轮宽度的年际间变异重建过去的气 候和环境变化,在全球变化研究中已被广泛应用 (Cook et al . ,1991)。随着研究理论和技术手段的改 进, 除了注意温度、降水等影响外, 对厄尔尼诺现象 紧密关联的南方涛动指数、冰川进退、径流与水位、 全球 $\mathrm{CO}_{2}$ 增温等 利用树木年轮将得到更多的再现 (吴祥定,1990)。研究的物种和区域范围也将进一 步扩大，不再仅仅局限于干旱半干旱的温带或高山 地区的一些树木, 甚至被埋藏的硅化木和炭化木的 
年轮宽度也不断被用于气候变化分析 (Briffa et al. ， 1990)。不同种类树木的生长对气候变化表现出不 同的响应特征 (Peterson \& Peterson,1994;Tardif \& Bergeron ,1997) , 也是决定末来气候变化条件下森林 物种组成变化的一个重要因素, 故物种的差别也将 会成为相关研究的一个主要方面 (侯爱敏等, 1999; Xiong et al. 2000)。另外, 有关利用树轮资料对气 候要素的重建工作及其对生态系统的影响已逐渐从 中小尺度上向空间大尺度上转移。空间上大尺度地 重建气候要素的过去变化, 是树轮宽度应用研究的 延伸,也是树木年轮气候学发展的必然方向 (邵雪 梅, 1997)。

但随着科学技术的进步, 新仪器设备的使用, 年 轮宽度不再是唯一的数据来源, 年轮密度及细胞结 构分析等正在成为研究中的新焦点(刘洪滨等, 1996)。许多研究证明, 年轮平均密度特别是晚材密 度特征相对于年轮宽度来说对气候变化更为敏感

(D'Arrigo et al. ,1996 ; Wiles et al .,1996)。Wimmer \& Grabner (1997) 则发现年轮中的树脂导管密度相 对于年轮宽度特征与气候的相关更为密切。Gindl 等 (2000) 还发现晚材的木质素和树轮宽度及最大密 度一样易受气候变化的影响。D'Arrigo 等 (1992) 在 研究北美森林树线上的白云杉与气候变化关系过程 中发现 树木年轮宽度与最大晚材密度虽然都是反 应树木生长状况的敏感指示器, 但它们对某些气候 因子的反应互不相同却又相互补充。如果能将它们 联合起来分析则能更充分地反映高海拔地带的气候 变化。Hughes 等利用树轮宽度结合密度资料成功地 重建了爱丁堡 $1721 \sim 1975$ 年间 7 8 月的温度 (Hughes et al . 1984) 和中国北中部自从 1600 年来 的降雨量 (Hughes et al , 1994)。更值得一提的是, 有关树轮纤维素碳同位素的研究表明，纤维素 $\delta{ }^{13} \mathrm{C}$ 可独立提供与树轮宽度和密度共存的气候信息 (Lipp et al. ,1996)。因此, 对它进行研究与树轮宽 度指数进行综合分析, 无疑更有利于我们更准确地 认识气候历史。陈拓等 (2000)利用新疆昭苏云杉树 轮纤维素 ${ }^{13} \mathrm{C}$ 与降水之间交互相关的函数关系, 恢复了近 300 年来的新疆昭苏地区的降水变化, 恢 复的系列与历史文献记录和树轮宽度指数所揭示的 历史降水有很好的对应性。

从现实的研究需要来看, 为减少树木年轮宽度 用于古气候研究中的不确定性, 今后有关树木年轮 对气候变化影响所引起的多方面响应特征综合起来 进行的研究将会进一步加强。在现代科技手段作为
坚强后盾的情况下, 各交叉学科的发展为这方面的 研究提供了更广阔的应用前景, 这一领域的研究也 将会为人类的发展和社会的进步做出不可低估的贡 献。

\section{参 考 文 献}

Akkemïk, Ü. 2000. Dendroclimatology of umbrella pine ( Pinus pine L. ) in Istanbul, Turkey. Tree-Ring Bulletin, 56: $17 \sim 20$.

Abrams, M. C., D. A. Orwig \& M. J. Dockry. 1997. Dendroecology and successional status of two contrasting old-growth oak forests in the Blue Ridge Mountains, USA. Canadian Journal of Forest Research, 27: $994 \sim 1002$.

Abrams, M. C., C. M. Ruffner \& T. A. Morgan. 1998. Treering responses to drought across species and contrasting sites in the ridge and valley of central Pennsylvania. Forest Science, 44: $550 \sim 558$.

Archambault, S. \& Y. Bergeron. 1992. An 802-year tree-ring chronology from Quebec boreal forest. Canadian Journal of Forest Research, 22: $674 \sim 682$.

Becker, M. 1989. The role of climate on present and past vitality of silver fir forests in the Vosges Mountains of northeastern France. Canadian Journal of Forest Research, 19: $1110 \sim 1117$.

Bhattarcharyya, A., V. C. LaMarche \& F. W. Telewski. 1988. Dendrochronological reconnaissance of the conifers of northwest Indian. Tree-Ring Bulletin, 48: $21 \sim 30$.

Borgaonkar, H. P., G. B. Pant \& K. R. Kumar. 1999. Treering chronologies from western Himalya and their dendroclimatic potential. International Association of Wood Anatomists, 20: 295 $\sim 309$.

Bräuning, A. 1999. Dendroclimatological potential of drought-sensitive tree stands in southern Tibet for the reconstruction of monsoonal activity. International Association of Wood Anatomists, 20: $325 \sim 338$.

Briffa, K. R., T. S. Bartholin, D. Eckstein, P. D. Jones, W. Karlén, F. H. Schweingruber \& P. Zetterberg. 1990. A 1400year tree-ring record of summer temperatures in Fennoscandia. Nature, 346: $434 \sim 439$.

Byram, G. M. \& W. T. Doolittle. 1950. A year of growth for a shortleaf pine. Ecology, 31: $27 \sim 35$.

Caritat, A., E. Gutierrez \& M. Molinas. 2000. Influence of weather on cork-ring width. Tree Physiology, 20: $893 \sim 900$.

Ceulemans, R. \& M. Mousseau. 1994. Effects of elevated atmospheric $\mathrm{CO}_{2}$ on wood plants. New phytologist, 127: $425 \sim 446$.

Chen, T. (陈拓), D. H. Qin(秦大河), J. F. Li(李江风), J. W. Ren(任贾文), X. H. Liu(刘晓宏) \& W. Z. Sun(孙维 贞). 2000. Study on climatic signification of fir tree-ring $\delta^{13} \mathrm{C}$ from Zhaosu Country of Xinjiang region, China. Journal of Glaciology and Geocryology (冰川冻土), 22: 347 352. (in Chinese with English abstract)

Cherubini, P., F. H. Schweingruber \& T. Forester. 1997. Morphology and ecological significance of intra-annual radial cracks in living conifers. Trees, 11: $216 \sim 222$.

Cook, E. R. 1985. A time series approach to tree-ring standardization. Ph. D. dissertation of University of Arizona, Tucson, AZ, USA.

Cook, E., T. Bird, M. Peterson, M. Barbetti, B. Buckley, R. D'Arrigo, R. Francey \& P. Tans. 1991. Climate change in Tasmania inferred from a 1089-year tree-ring chronology of Huon pine. Science, 253: $1266 \sim 1268$.

D'Arrigo, R. D., G. C. Jacoby \& R. M. Free. 1992. Tree-ring width and maximum latewood density at the North American tree line: parameters of climatic change. Canadian Journal of Forest Research, 22: $1290 \sim 1296$. 
D'Arrigo, R. D., E. R. Cook \& G. C. Jacoby. 1996. Annual to decadal-scale variations in northwest Atlantic sector temperatures inferred from Labrador tree rings. Canadian Journal of Forest Research, 26: $143 \sim 148$.

D'Arrigo, R. D. \& G. C. Jacoby. 1999. Northern North American tree-ring evidence for regional temperature changes after major volcanic events. Climatic Change, 41: 1 $\sim 15$.

Douglass, A. E. 1914. A method for estimating rainfall by the growth of trees. In: Huntington, E. ed. The climatic factors as illustrated in arid America. Lancaster, Pennsylvania: Carnegie Institute of Washington Publication. 192: 101 121 .

Douglass, A. E. 1920. Evidence of climatic effects in the annual rings of trees. Ecology, 1:24 32 .

Edwards, N. T. \& P. J. Hanson. 1996. Stem respiration in closed canopy upland oak-forest. Tree Physiology, 16: $433 \sim 439$.

Emanuel, W. R., H. H. Shugart \& M. L. Stevenson. 1985. Climatic change and the broad-scale distribution of terrestrial ecosystem complexes. Climatic Change, 7: 29 43.

Fritts, H. C., D. G. Smith \& M. A. Stokes. 1965a. The biological model for paleoclimatic interpretation of Mesa Verde tree-ring series. American Antiquity, 31:101 121 .

Fritts, H. C., D. G. Smith, J. W. Cardis \& C. A. Budelsky. 1965b. Tree-ring characteristics along a vegetation gradient in northern Arizona. Ecology, 46: $393 \sim 401$.

Fritts, H. C. 1966. Growth-rings of trees: their correlation with climate. Science, 154: $973 \sim 980$.

Fritts, H. C. 1969. Bristlecone pine in the White Mountains of California: growth and ring-width characteristics. In: Papers of the Laboratory of Tree-ring Research, University of Arizona, No. 4. Tucson: The University of Arizona Press.

Fritts, H. C. 1976. Tree rings and climate. London: Academic Press. 534.

Fritts, H. C. 1991. Rconstuction large-scale climate patterns from tree-ring data. Tucson: The University of Arizona Press.

Fritts, H. C. 1992. Dendrochrological modeling of the effects of climatic change on tree-ring width chronologies from the Chaco Canyon area, southwestern United States. Tree-Ring Bulletin, 52: $31 \sim 58$.

Gindl, W., M. Grabner \& R. Wimmer. 2000. The influence of temperature on latewood lignin content in treeline Norway spruce compared with maximum density and ring width. Trees, 14: 409 $\sim 414$.

Graybill, D. A. \& S. G. Shiyatov. 1989. A 1009 year tree-ring reconstruction of mean June-July temperature deviations in the Polar Urals. In: Tree-Ring Bulletin, 1997, special issue (Reprinted from Nobel, R. D., J. L. Martin \& K. F. Jensen eds. 1989. Symposium on air pollution effects of vegetation. USDA forest service, northwestern forest experiment station. 37 42)

Graumlich, L. J. 1991. Subalpine tree growth, climate, and increasing $\mathrm{CO}_{2}$ : an assessment of recent growth trends. Ecology, 72: $1 \sim 11$.

Graumlich, L. J. 1993. A 1000-year record of temperature and precipitation in Sierra Nevada. Quaternary Research, 39: 249 255 .

Green, K. \& R. Wright. 1977. Field response of photosynthesis to $\mathrm{CO}_{2}$ enhancement in ponderosa pine. Ecology, 58: 687 692 .

Groisman, P. Y., T. R. Karl \& R. W. Knight. 1994. Observed impact of snow cover on the heat balance and the rise of continental spring temperatures. Science, 263: $198 \sim 200$.

Gutiérrez, E. 1991. Climate tree-growth relationships for Pinus uncinata Ram. in the Spanish pre-Pyrenees. Acta Oecoligia, 12: $213 \sim 225$.

Holmes, R. L., C .W. Stockton \& V. C. LaMaarche Jr. 1979. Extension of river flow records in Argentina from living tree-ring chronologies. Water Resources Bulletin, 15:1081 1085 .

Hou, A. M. (侯爱敏) , S. L. Peng(彭少麟) \& G. Y. Zhou(周 国逸 ). 1999. The study of the reconstruction of tree rings to the climate change and its application. Ecologic Science (生态科 学), 18:16 23. (in Chinese with English abstract)

Hughes, M. K., P. M. Kelly, J. R. Pilcher \& V. C. LaMarcher Jr. 1982. Climate from tree rings. Cambridge: Cambridge University Press.

Hughes, M. K., F. H. Schweingruber, D. Cartwright \& P. M. Kelly. 1984. July-August temperature at Edinburgh between 1721 and 1975 from tree-ring density and width data. Nature, 308: $341 \sim 344$.

Hughes, M. K., X. D. Wu, X. M. Shao \& G. M. Garfin. 1994. A preliminary reconstruction of rainfall in North-central China since A. D. 1600 from tree-ring density and width. Quaternary Research, 42: $88 \sim 99$.

Jacoby, G. C. \& R. D. D' Arrigo. 1997. Tree rings, carbon dioxide, and climate change. Proceedings of the National Academy of Sciences, USA, 94: 8350 8353.

Jacoby, G. C., R. D. D'Arrigo \& T. Davaajamts. 1997. Mongolian tree rings and 20th-century warming. Science, 273: $771 \sim$ 773 .

Jenkins, M. A. \& S. G. Pallardy. 1995. The influence of drought on red oak group species growth and mortality in the Missiuri Ozarks. Canadian Journal of Forest Research, 25: 1119 1127.

Jiang, G. M. (蒋高明), X. G. Han(韩兴国)\& G. H. Lin(林 光辉). 1997. Response of plant growth to elevated $\left[\mathrm{CO}_{2}\right]: \mathrm{a}$ review on the chief method sand basic conclusions based on experiments in the external countries in past decade. Acta Phytoecologica Sinica (植物生态学报), 21:489 502. (in Chinese with English abstract)

Johnson, E. A. \& G. I. Fryer. 1989. Population dynamics in lodgepole pine-Engelmann spruce forests. Ecology, 70: 1335 1345 .

Jones, P. D., K. R. Briffa \& J. R. Pilcher. 1984. River-flow reconstruction from tree rings in Southern Britain. Journal of Climatology, 4: $461 \sim 472$.

Kang, X. C. (康兴成), L. J. Graumlich \& P. Scheppard. 1997. The last 1835 years climates inferred from tree ring records in Dulan region, Qinghai, China. Quaternary Sciences (第四纪研 究), 1: 70 75. (in Chinese with English abstract)

Kienast, F., F. H. Schweingruber, O. U. Bräker \& E. Schär. 1987. Tree-ring studies on conifers along gradients and the potential of single-year analyses. Canadian Journal of Forest Research, 17: $687 \sim 696$.

Kienast, F. \& R. J. Luxmoore. 1988. Tree-ring analysis and conifer growth responses to increased atmospheric $\mathrm{CO}_{2}$ levels. Oecologia, 76: $487 \sim 495$.

Körner, C. \& M. Diemer. 1987. In situ photosynthetic response to light, temperature and carbon dioxide in herbaceous plants from low and high latitude. Functional Ecology, 1: 179 194 .

LaDeau, S. L. \& J. S. Clark. 2001. Rising $\mathrm{CO}_{2}$ levels and the fecundity of forest trees. Science, 292: $95 \sim 98$.

LaMarche, V. C. Jr. 1974a. Paleoclimatic inferences from long tree-ring records. Science (Washington), 183: $1043 \sim 1048$.

LaMarche, V. C. Jr. 1974b. Frequency-dependent relationships between tree-ring series along an ecological gradient and some dendroclimatic implications. Tree-Ring Bulletin. 34:1 20.

LaMarche, V. C. Jr., D. A. Graybill, H. C. Fritts \& M. R. Rose. 1984a. Increasing atmospheric and carbon dioxide: tree ring evidence for growth enhancement in annual vegetation. Science, 225: $1019 \sim 1021$.

LaMarche, V. C. Jr. \& K. K. Hirschboeck. 1984b. Frost rings in trees as records of major volcanic eruptions. Nature, 307: 121 $\sim 126$. 
Lan, T. (兰涛)，B．Xia(夏冰)\& S. A. He(贺善安)． 1994. Tree ring analysis on relation of Pinus massoniana growth to climate factors. Chinese Journal of Applied Ecology (应用生态学 报), 5: $422 \sim 424$. (in Chinese with English abstract)

Larsen, C. P. S. \& G. M. MacDonald. 1995. Relations between tree-ring widths, climate, and annual area burned in the boreal forest of Alberta. Canadian Journal of Forest Research, 25: 1746 $\sim 1755$.

Lemon, E. R. 1983. $\mathrm{CO}_{2}$ and plants: the response of plants to rising levels of atmospheric carbon dioxide. Boulder, Colorado: Westview Press.

Li, J. F. (李江风) \& Y. J. Yuan (袁玉江). 1984. Affect of snow in the tree-ring. Journal of Xinjiang University (Nature Science) (新疆大学学报 (自然科学版) ), 2: 85 92. (in Chinese with English abstract)

Li，J. F. (李江风),Y.J. Yuan (袁玉江) \& X. Y. You (由希 尧). 1997. 360 years' runoff reconstruction in Urumqi river basin using tree rings. Quaternary Sciences (第四纪研究), 2: $131 \sim 146$. (in Chinese with English abstract)

Li, J. F. (李江风), Y. J. Yuan (袁玉江) \& X. Y. You (由希 尧). 2000. The study on tree ring hydrology and its applications. Beijing: Science Press. (in Chinese)

Liang, E. Y., X. M. Shao, Y. X. Hu \& J. X. Lin. 2001. Dendroclimatic evaluation of climate-growth relationships of Meyer spruce (Picea meyeri) on a sandy substrate in semi-arid grassland, north China. Trees, 15: 230 235 .

Liang, E. Y. (梁尔源). 2001. A dendroecological study of Picea meyeri. Ph. D. dissertation of Institute of Botany, the Chinese Academy of Sciences. (in Chinese with English abstract)

Lipp, J., P. Trimborn, T. W. D. Edwards, W. Graf \& B. Becker. 1996. Climate signals in ${ }^{2} \mathrm{H}$ and ${ }^{13} \mathrm{C}$ chronology (18821989) from tree rings of spruce (Picea abies L.), schussbach forest, Germany. In: Dean, J. S., M. Meko \& T. W. Swetnam eds. Tree rings, environment and humanity. Tucson: University of Arizona Press. $603 \sim 610$.

Liu, H. B. (刘洪滨), X. D. Wu (吴祥定) \& X. M. Shao (邵 雪梅). 1996. A preliminary study on climate change research during historical time using image analysis of tree ring in Kangding area, Sichuan province. Geographical Research (地理 研究), 15: $44 \sim 51$. (in Chinese with English abstract)

Liu, H. B. (刘洪滨) \& X. M. Shao (邵雪梅). 2000. Reconstruction of early-spring temperature at Zhen' an from 1755 using tree ring chronology. Acta Meteorologica Sinica (气象学报), 58: $223 \sim 233$. (in Chinese with English abstract)

Mäkinen, H. , P. Nöjd \& K. Mielikäinen. 2001. Climatic signal in annual growth variation in damaged and healthy stands of Norway spruce (Picea abies (L) Karst.) in south Finland. Trees, 15: $177 \sim 185$.

McClenahen, J. R. 1995. Potential dendroecological approaches to understanding forest decline. In : Lewis, T. E. ed. Tree rings as indicators of ecosystem health. Florida: CRC Press, $59 \sim 79$.

Meko, D. M. 1981. Applications of Box-Jenkins Methods of time series analysis to the reconstruction of drought from tree rings. Ph. D. dissertation of University of Arizona, Tucson, AZ, USA.

Mooney, H. A., R. D. Wright \& B. R. Strain. 1964. The gas exchange capacity of plants in relation to vegetation zonation in the White Mountains of California. American Midland Naturalist, 72: $281 \sim 297$.

Nicolussi, K., S. Bortenschlager \& C. Korner. 1995. Increase in tree-ring width in subalpine Pinus cembra from the central Alps that may be $\mathrm{CO}_{2}$-related. Trees, 9: $181 \sim 189$.

Nowack, G. J. \& M. D. Abrams. 1997. Radial-growth averaging criteria for reconstructing disturbance histories from presettlementorigin oaks. Ecological Monographs, 67: $225 \sim 249$.

Oberhuber, W ., M. Stumböck \& W. Kofler. 1998. Climate-tree- growth relationships of Scots pine stands (Pinus sylvestris L.) exposed to soil dryness. Trees, 13: 19 27 .

Overpeck, J., K. Hughen, D. Hardy, R. Bradley, R. Case, M. Douglas, B. Finney, K. Gajewski, G. Jacoby, A. Jennings, S. Lamoureux, A. Lasca, G. MacDonald, J. Moore, M. Retelle, S. Smith, A. Wolfe \& G. Zielinski. 1997. Arctic environmental change of the last four centuries. Science, 278: 1251 $\sim 1256$.

Parker, M. L. \& W. E. S. Henoch. 1971. The use of Engelman spruce latewood density for endrochronological purposes. Canadian Journal of Forest Research, 1: $90 \sim 98$.

Peterson, D. W. \& D. L. Peterson. 1994. Effects of climates on radial growth of subalpine conifers in the North Cascade Mountains. Canadian Journal of Forest Research, 24: $1921 \sim 1932$.

Polge, H. 1970. The use of X-ray densitometric methods in dendrochronology. Tree-Ring Bulletin, 30: 1 10.

Rogers, H. H., J. F. Thomas \& G. E. Bingham. 1983. Response of agronomic and forest species to elevated atmospheric carbon dioxide. Science, 220: 428 429 .

Rolland, C. 1993. Tree-ring and climate relationships for Abies al$b a$ in the internal Alps. Tree-Ring Bulletin, 53: 1 11.

Savva, Y., F. Schweingruber, L. Milyyutin \& E. Vaganov. 2002. Genetic and environmental signals in tree rings from different provenances of Pinus sylvestris L. planted in the southern taiga, central Siberia. Trees, 16:313 324 .

Schweingruber, F. H. 1988. Tree rings: basics and applications of dendrochronology. Dordrecht, Netherlands: Kluwer Academic Publisher.

Schweingruber, F. H. 1996. Tree rings and environment: dendroecology. Berne: Paul Haupt Publishers. $21 \sim 39$.

Shao, X. M. (邵雪梅) \& X. D. Wu. (吴祥定) . 1994. Tree-ring chronologies for Pinus armandi Franch from Huashan, China. Acta Geographica Sinica (地理学报), 2: 174 181. (in Chinese with English abstract)

Shao, X. M. (鄂雪梅). 1997. Advancements in dendrochronology. Quaternary Sciences (第四纪研究), 3: $265 \sim 271$. (in Chinese with English abstract)

Shao, X. M. (邵雪梅) \& J. M. Fan(范金梅) . 1999. Past climate on west Sichuan Plateau as reconstructed from ring-width of dragon spruce. Quaternary Sciences (第四纪研究), 1: 81 89. (in Chinese with English abstract)

Sheppard, P. R., L. J. Graumlich \& L. E. Conkey. 1996. Reflected-light image analysis of conifer tree rings for reconstructing climate. Holocene, 6: $62 \sim 68$.

Solomon, A. M. 1986. Transient response of forests to $\mathrm{CO}_{2}$-induced climate change: simulation modeling experiments in eastern North America. Oecologia (Berlin), 68: $567 \sim 579$.

Stahle, D. W. , M. K. Cleaveland \& J. G. Hehr. 1985. A 450year drought reconstruction for Arkansas, United States. Nature, 316: $530 \sim 532$.

Stahle, D. W., M. K. Cleaveland, D. B. Blanton, M. D. Therrell \& D. A. Gay. 1998. The lost colony and Jamestown droughts. Science, 280: $564 \sim 567$.

Steele, B. \& C. Fiedler. 1996. Kalman filter analysis of growthclimate relations in old-growth Pondersa pine/Douglas-fir stands. In: Dean, J. S., D. M. Meko \& T. W. Swetnam eds. Tree rings, environment and humanity. Tucson: Department of Geoscience, the University of Arizona. $303 \sim 314$.

Swetnam, T. W., 1993. Fire history and climate change in giant Sequoia Groves. Science, 262: $885 \sim 889$.

Szeicz, J. M. \& G. M. MacDonald. 1994. Aged-dependent growth responses of subartic white spruce to climate. Canadian Journal of Forest Research, 24:120 132 .

Szeicz, J. M. \& G. M. MacDonald. 1996. A 930-year ring-width chronology from moisture-sensitive white spruce (Picea glauca Moench) in the northwestern Canada. The Holocene, 6: 345 
351 .

Tardif, J. \& Y. Bergeron. 1997. Comparative dendroclimatological analysis of two blank ash and two white cedar population from contrasting sites in the lake Duparquet region, northwestern Quebec. Canadian Journal of Forest Research, 27: $108 \sim 116$.

Tessier L. 1989. Spatio-temporal analysis of climate-tree ring relationships. New Phytologist., 11:517 529.

Thetford, R. D., D. D’Arrigo \& G. C. Jacoby. 1991. An image analysis for determining dendsitomotric and ring-width time series. Canadian Journal of Forest Research, 21: $544 \sim 549$.

Van Deusen, P. C. \& G. A. Reams. 1996. Bayesian Procedures for reconstructing past climate. In: Dean, J. S., D. M. Meko \& T. W. Swetnam eds. Tree rings, environment and humanity. Tucson: Department of Geoscience, the University of Arizona. $335 \sim 339$.

Vaganov, E. A. 1990. Method for forecasting grain yield with the aid of dendrochronological data. Soviet Journal of Ecology, 20 : $139 \sim 146$

Vaganov, E. A. \& A. Kachaev. 1992. Construction and usage of a complex model to illustrate the relationship between tree increment and wheat crop yield. Lundqua Report, 34:327 $\sim 330$.

Vaganov, E. A., M. K. Hughes, A. V. Kirdyanov, F. H. Schweingruber \& P. P. Silkin. 1999. Influence of snowfall and melt timing on tree growth in subarctic Eurasia. Nature, 400: $149 \sim 151$

Veblen, T. T., K. S. Handley \& M. S. Reid. 1991a. Disturbance and stand development of a Colorado subalpine forest. Journal of Biogeography, 18:707 716 .

Veblen, T. T., K. S. Handley, M. S. Reid \& A. J. Rebertus. 1991b. Methods of detecting past spruce beetle outbreaks in Rocky Mountain subalpine forests. Canadian Journal of Forest Research, 21:242 254.

Wang, M. (王沝), S. J. Bai(白淑菊), D. L. Tao(陶大立) \& J. P. Shan(单建平). 1995. Effect of rise in air-temperature on tree ring growth of forest on Changbai Moutain. Chinese Journal of Applied Ecology (应用生态学报), 6:128 132. (in Chinese with English abstract)

Wiles, G. C., R. D. D'Arrigo \& G. C. Jacoby. 1996. Temperature changes along the Gulf of Alaska and the Pacific northwest coast modeled from coastal tree rings. Canadian Journal of Forest Research, 26: $474 \sim 481$.

Wimmer, R. \& M. Grabner. 1997. Effects of climate on vertical resin duct density and radial growth of Norway spruce (Picea abies (L. ) Karst.). Trees, 11: 271 276.
Wimmer, R. \& M. Grabner. 2000. A comparison of tree-ring features in Picea abies as correlated with climate. International Association of Wood Anatomists, 21: $403 \sim 416$.

Woodwell, G. M., J. E Hobbie., R. A. Houghton, J. M. Melillo, B. Moore, B. J. Peterson \& G. R. Shaver. 1983. Global deforestation: contribution to atmospheric carbon dioxide. Science, 222: $1081 \sim 1086$.

Wu, X. D. (吴祥定). 1990. Tree rings and climate change. Beijing: Meteorology Press. (in Chinese)

Xia, B. (夏冰), S. A. He(贺善安) \& T. Lan (兰涛). 1994. A preliminary study on the relationships between tree rings of Pinus massoniana Lamb. and local crop yield. Journal of Plant Resources and Environment (植物资源与环境), 3:27 32. (in Chinese with English abstract)

Xia, B. (夏冰), F. Deng (邓飞), K. Zhou (周康), Q. Wang (王谦) \& X. Dong (董旭). 2001. A case study on the verification of density of forest disturbance through tree-ring analysis. Journal of Plant Resources and Environment (植物资源与环境 学报), 10: 1 6. (in Chinese with English abstract)

Xie, K. Q. (谢昆青) \& Z. Y. Li (李志尧). 2000. Method of scanning image analysis for tree-ring research and its application in environmental change. Quaternary Sciences (第四纪研究), 20: $259 \sim 269$. (in Chinese with English abstract)

Xiong, L. N. Okada \& T. Fujiwara. 2000. The dendrochrnological potential of the species in the Three Gorges Reservoir region of China. International Association of Wood Anatomists, 21: 181 196.

Yamaguchi, D. K., B. F. Atwater, D. E. Buunker, B. E. Benson \& M. S. Reid. 1997. Tree-ring dating the 1700 Cascadia earthquake. Nature, 389: 822 823.

Yuan, Y. J. (袁玉江). 1987. Forecasting climatic output of spring-wheat in Altay county by using of tree-ring chronologies. Bimonthly of Xinjiang Meteorology (新疆气象), 10:16 19 . (in Chinese with English abstract)

Yuan, Y. J. (袁玉江) \& J. F. Li (李江风). 1999. Reconstruction and analysis of 450 years' winter temperature series in the Ürümqi river source of Tianshan Mountains. Journal of Glaciology and Geocryology (冰川冻土), 1:64 70. (in Chinese with English abstract)

Zhang, Z. H. (张志华), X. D. Wu (吴祥定) \& J. Li (李骥). 1996. Utilizing tree ring chronologies to reconstruct 300-year drought days in eastern Tianshan Mountains. Quarterly Journal of Applied Meteorology (应用气象学报), 7: 53 60. (in Chinese with English abstract) 